13. Emanuel, E. J., Wendler, D. \& Grady, C. J. Am. Med. Assoc. 283, 2701-2711 (2000)

14. Taubenberger, J. K., Morens, D. M. \& Fauci, A. S. J. Am. Med. Assoc. 297, 2025-2027 (2007)

15. Wosik, J. et al. J. Am. Med. Inform. Assoc. 27, 957-962 (2020)

16. Kim, S. Y. H. \& Grady, C. Neurology $94,1007-1008$ (2020).
Acknowledgements

The authors thank the members of the NIH BRAIN Initiative Neuroethics Working Group, meeting presenters (M. Franceschini, L. Hochberg and S. Sheth) and the participants and organizers of the neuroethics-focused session at the BRAIN Initiative Investigators Meeting. This research was supported in part by the Intramural Research
Program of the NIH. The views expressed are the authors' own and do not necessarily reflect those of the National Institutes of Health, the Department of Health and Human Services, or the United States government.

Competing interests

The authors declare no competing interests.

\title{
To tackle diabetes, science and health systems must take into account social context
}

\author{
An increasing amount of publications are recognizing that a person's risk of diabetes and diabetes outcomes are \\ influenced largely by social determinants of health. This renewed understanding of disease should influence health \\ provision and diabetes research, but will it?
}

\section{Jacqueline A. Seiglie, Devaki Nambiar, David Beran and J. Jaime Miranda}

\begin{abstract}
$\mathrm{n}$ a year marked by the centenary of the discovery of insulin ${ }^{1}$ and outstanding scientific progress in the therapeutic management of diabetes mellitus ${ }^{2,3}$, these advances have been contrasted by the sobering disparities that, although present before the COVID-19 pandemic, have been unmasked by this crisis for people living with diabetes. While diabetes is now well recognized as an important biological risk factor for poor COVID-19 outcomes, the disproportionate impact of this pandemic on socially vulnerable people with diabetes has laid bare the profound importance of the social determinants of health in the tackling of health inequalities ${ }^{4,5}$. These disparities are particularly alarming when one considers that among the nearly half-billion people with diabetes globally, three out of every four people with diabetes live in low- and middle-income countries (LMICs) ${ }^{6}$. The renewed attention on the social determinants of health in relation to diabetes in the wake of the COVID-19 pandemic has revealed the sheer complexity of tackling health inequalities and the inadequacy of existing metrics, frameworks and approaches ${ }^{7}$. The question is: amid the astounding scientific progress accomplished in the management of diabetes, what does a social-determinants framework add to the field, particularly in LMICs?

It has long been recognized that health is strongly influenced by social determinantsthe conditions in which people are born, grow, live, work, and age, including the health system ${ }^{8}$. In fact, the concept of the social determinants of health was woven
\end{abstract} into the very foundations of modern public health, which at the turn of the 19th century inspired pioneers such as Rudolf Virchow to posit that social medicine was inextricably intertwined with the politics of social justice $^{9,10}$. Further evidence on the strong linkage between the social determinants and their impact on disease emerged throughout the 20th century ${ }^{11}$ and, in 2008, culminated in the report of the World Health Organization's Commission on the Social Determinants of Health ${ }^{8}$, which called for a global movement to recognize their importance in the tackling of health inequalities, with the goal of closing the health gap within a generation. Yet more than a decade since that call for action was put forth, the COVID-19 crisis has abruptly highlighted the lack of progress achieved in tackling inequalities, making visible the linkages and the gross weaknesses of living conditions, health and well-being, and the impact of this on multiple facets of long-term chronic conditions ${ }^{12,13}$.

In their timely article 'Social determinants of health and diabetes: a scientific review, published in Diabetes $\mathrm{Care}^{4}$, the authors provide an overview of key definitions and social-determinant frameworks of diabetes and outline detailed recommendations for their implementation into community sectors, diabetes research and clinical practice in the USA. The review builds on pragmatic scientific and consensus statements published before the COVID-19 pandemic ${ }^{14,15}$ and categorizes social determinants of diabetes into the following five domains: socioeconomic status; neighborhood and physical environment; food environment; healthcare; and social context ${ }^{4}$. Each of these domains encompass aspects of life circumstances that influence how a person's risk of diabetes and diabetes outcomes are shaped-a phenomenon that occurs largely outside of the health system $^{16}$. While there is robust evidence to support this framework, much of this evidence has been generated in high-income contexts. Less is known about the social determinants of diabetes in LMICs, which are highly heterogeneous and may be subject to additional societal pressures, such as socio-political and protracted conflicts ${ }^{17}$, environmental pollutants ${ }^{18}$, and corporate and commercial power ${ }^{19,20}$, as well as constrained health systems with limited capacity to manage chronic conditions that rely on continuity of care ${ }^{21}$.

Whereas much of the scientific progress in diabetes has gravitated toward the domain of pharmacological interventions, diabetes 'exists' within a much broader context, with factors that influence its development throughout the life course. For instance, an adverse intrauterine and postnatal environment may influence the development of insulin resistance and the onset of metabolic disease later in life $^{22}$. When these early life disadvantages are coupled with social and commercial determinants that catalyze the development of overweight and obesity ${ }^{19}$, the cumulative burden of these life-course circumstances, which disproportionately affect vulnerable segments of the population, can be difficult to overcome even within equitable health systems. Notably, solutions for mitigating the risk of diabetes may very well lie outside the sphere of health systems, as 
demonstrated by a housing experiment showing that the opportunity to move to a neighborhood with a lower poverty rate was associated with a lower prevalence of severe obesity and poorly controlled diabetes ${ }^{16}$. As contended by others, "a diagnosis is rarely a solution to problems caused by poverty and inequality" ${ }^{23}$. Furthermore, there is a strong socioeconomic patterning of diabetes and other non-communicable diseases at the population level, whose rise in LMICs is occurring in the context of a complex interplay of epidemiological, demographic and nutritional transitions $\mathrm{s}^{24,25}$. Within this framework, diabetes and other non-communicable diseases are posited to rise initially among affluent groups before shifting to groups of low socioeconomic status $^{26,27}$. It is critical to recognize this socioeconomic patterning of diabetes, ideally before the burden of diabetes shifts among populations facing socioeconomic and other vulnerabilities, which requires that additional obstacles, manifold in quantity and complexity, be overcome.

While the social-determinants framework is fundamental for understanding elements largely outside of the health system that contribute to the risk of diabetes and its outcomes, insightful medical anthropologists have long proposed the broader framework of 'syndemic' ('synergistic epidemic') theory ${ }^{28,29}$. A syndemic refers to the clustering of two or more diseases within a population that contributes to, and results from, persistent social and economic inequalities ${ }^{28,30}-\mathrm{a}$ concept that is particularly relevant in the context of the intersection between COVID-19 and diabetes and that highlights social determinants as core components of the effort to eradicate disparities ${ }^{30}$. Indeed, it has been proposed that in tackling COVID-19 and its interaction with non-communicable diseases, "the syndemic nature of the threat we face means that a more nuanced approach is needed if we are to protect the health of our communities"30. A 'syndemic lens' can both lead to a deeper understanding of the reasons for the clustering of diabetes in certain populations and inform targeted policies to address broader structural and political forces that impact both the development of diabetes and diabetes-related outcomes ${ }^{31}$. This framework requires a nuanced understanding of context-dependent factors that influence disease development and progression, as well as investment in research that can tackle the biosocial complexities of diabetes $28,29,32$.

The strengthening of health systems for the provision of diabetes care in LMICs is critical. However, the development of health systems that solely prioritize short-term care delivery, relying on patient-provider interaction or health-system inputs, is likely to fall short in tackling both the rising burden of diabetes and its associated disparities. This core challenge, of recognizing the limitations of the current models of care, is poignantly captured by this reflection on patients' real-world experience of living with type 2 diabetes in Peru: "a patient's agency to manage their [type 2 diabetes] is affected by a multiplicity of factors acting together: the burden of treatment, the chronicity of poverty, the immediate social context, the deficiencies in the health system, as well as the financial burden of dietary change and medication adherence... the interaction among all these factors in an individual's vulnerability to ill-health have been recognized by critical medical anthropologists who developed the syndemics framework to acknowledge the role of broader political, economic, and social structures in the individual lives of people, their health, and responses to health" ${ }^{\text {"33 }}$.

How can the COVID-19 crisis be leveraged to revisit the approach to diabetes care in LMICs through a syndemic framework? Diabetes has been long considered a suitable 'tracer' condition of international benchmarking of health systems, since it is well defined, fairly easy to diagnose and common ${ }^{34}$. Given the enormous and rapidly growing prevalence of diabetes in LMICs, as well as its social patterning, we argue that diabetes is also a suitable tracer of health disparities ${ }^{35}$. Through its recognition as such, the approach to understanding the growing burden of diabetes in LMICs can be broadened beyond its biology and within its tightly linked social, economic and structural context ${ }^{29,32}$.

The publication of scientific statements on the importance of social-determinant frameworks is a great step forward ${ }^{4,14,15}$. However, will this translate into research efforts and changes in this arena beyond the exploration of disparities in high-income contexts? The incorporation of a social-determinants framework will require both a conceptual shift in the collective approach to the prevention and management of diabetes, beyond pathophysiology and pharmacotherapy, and concomitant investment in upstream factors as an integral aspect of the diabetes field. Funders, governments, the private sector and researchers should consider the critical importance of the social determinants of diabetes as the world moves toward the 'new normal' after COVID-19, to ensure a more equitable society in which health is not vitiated by underlying social and economic factors. The centenary of the discovery of insulin is a reminder not only of the impact science and medicine can have on health but also that if governments and civil society, including academia, do not play a role in addressing disparities, advances will be sequestered among the privileged.

And we return to our question: what does a social-determinants 'lens' add to the field, particularly in the LMIC context? The multi-faceted challenges imposed by COVID-19 and the neglected rising burden of diabetes in LMICs present a key opportunity for remembering the caution and the bold call to action put forward by the World Health Organization's Commission on the Social Determinants of Health in 2008: social injustice is killing people on a grand scale, and the health gap must be closed within a generation. It follows that 'building back better and fairer' ${ }^{36}$ will require rather more than advances in pharmacotherapy and technologies. Life course, the whole of society and intergenerational approaches ought to guide responses, attempting to tackle multiple drivers at once. Indeed, contending with the global, national and local legacies of power (asymmetries) lies at the heart of addressing diabetes-and the ailing health systems and societies its burden reflects-in the coming decades.

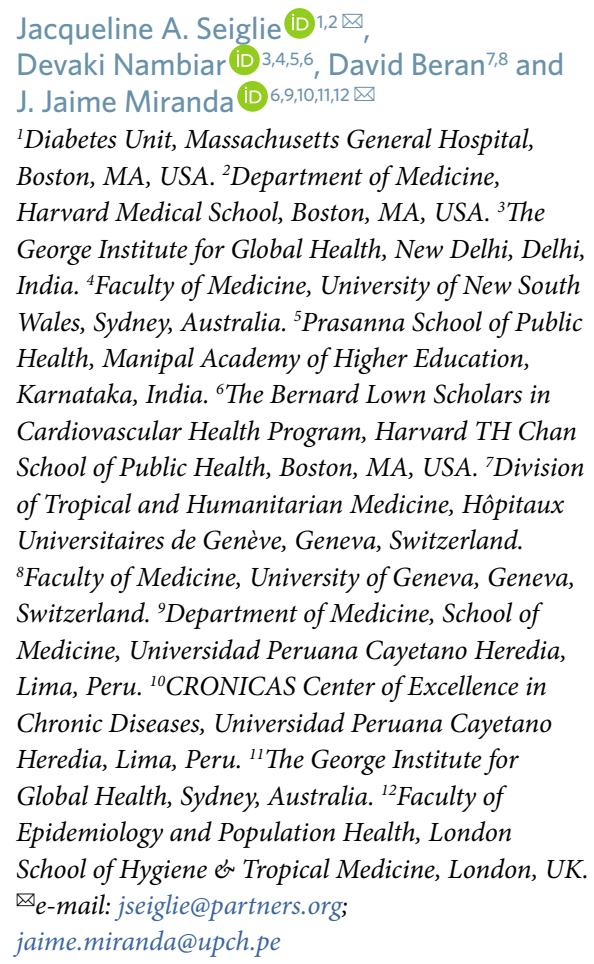

Published online: 1 February 2021 https://doi.org/10.1038/s41591-021-01231-X

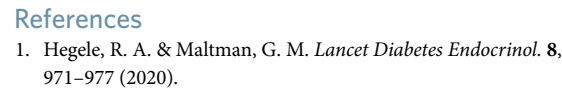


2. Sheahan, K. H., Wahlberg, E. A. \& Gilbert, M. P. Postgrad. Med. J. 96, 156-161 (2020).

3. Zannad, F. et al. Lancet 396, 819-829 (2020).

4. Hill-Briggs, F. et al. Diabetes Care https://doi.org/10.2337/dci200053 (2021).

5. Sosa-Rubí, S.G. et al. Diabetes Care https://doi.org/10.2337/dc20 2192 (2020).

6. International Diabetes Federation. https://www.diabetesatlas.org/ en/resources/ (accessed 18 December 2019).

7. Gianella, C., Gideon, J. \& Romero, M. J. Can. J. Dev. Stud. https:// doi.org/10.1080/02255189.2020.1843009 (2020).

8. World Health Organization. https://www.who.int/ publications-detail-redirect/WHO-IER-CSDH-08.1 (2008).

9. Nambiar, D. \& Muralidharan, A. (eds.) The Social Determinants of Health in India: Concepts, Processes, and Indicators https://doi. org/10.1007/978-981-10-5999-5 (Springer Singapore, 2017).

10. Reese, D. M. West. J. Med. 169, 105-108 (1998).

11. Marmot, M. G., Rose, G., Shipley, M. \& Hamilton, P. J. J. Epidemiol. Community Health 32, 244-249 (1978).

12. Nambiar, D., Bhaumik, S., Pal, A. \& Ved, R. BMC Health Serv. Res. 20, 1077 (2020).
13. Pesantes, M. A. et al. Rev. Peru. Med. Exp. Salud Publica 37, 541-546 (2020).

14. Hill, J. O. et al. Diabetes Care 36, 2430-2439 (2013).

15. Thornton, P. L. et al. Ann. NY Acad. Sci. 1461, 5-24 (2020).

16. Ludwig, J. et al. N. Engl. J. Med. 365, 1509-1519 (2011).

17. Boulle, P., Kehlenbrink, S., Smith, J., Beran, D. \& Jobanputra, K. Lancet Diabetes Endocrinol. 7, 648-656 (2019).

18. Liu, F. et al. Environ. Pollut. 252, 1235-1245 (2019).

19. McKee, M. \& Stuckler, D. Am. J. Public Health 108, 1167-1170 (2018).

20. de Lacy-Vawdon, C. \& Livingstone, C. BMC Public Health 20 , 1022 (2020).

21. Beran, D. Curr. Diab. Rep. 15, 20 (2015).

22. Lawlor, D. A., Davey Smith, G. \& Ebrahim, S. Diabetes Care 26, 97-103 (2003).

23. Burgess, R. Nature https://doi.org/10.1038/d41586-020-01313-9 (2020).

24. Popkin, B. M. Public Health Nutr. 1, 5-21 (1998).

25. Omran, A. R. Milbank Mem. Fund Q. 49, 509-538 (1971).

26. Jiwani, S. S. et al. Lancet Glob. Health 7, e1644-e1654 (2019).

27. Jaacks, L. M. et al. Lancet Diabetes Endocrinol. 7, 231-240

(2019).
28. Singer, M., Bulled, N., Ostrach, B. \& Mendenhall, E. Lancet 389, 941-950 (2017)

29. Mendenhall, E., Kohrt, B. A., Norris, S. A., Ndetei, D. \& Prabhakaran, D. Lancet 389, 951-963 (2017).

30. Horton, R. Lancet 396, 874 (2020).

31. Mendenhall, E. Lancet 396, 1731 (2020).

32. Saxena, A. \& Mendenhall, E. Soc. Sci. Med. https://doi. org/10.1016/j.socscimed.2020.113503 (2020).

33. Pesantes, M. A., Tetens, A., Valle, A. D. \& Miranda, J. J. Hum. Organ. 78, 85-96 (2019).

34. Nolte, E., Bain, C. \& McKee, M. Diabetes Care 29 1007-1011 (2006)

35. Taype-Rondan, A., Lazo-Porras, M., Moscoso-Porras, M., Moreano-Sáenz, M. \& Miranda, J. J. Br. J. Gen. Pract. 66, 197-197 (2016)

36. Marmot, M. The BMJ Opinion https://blogs.bmj. com/bmj/2020/12/15/michael-marmot-post-covid19-we-must-build-back-fairer/ (15 December 2020).

Competing interests

The authors declare no competing interests. 